\title{
Research on Power System Reliability Assessment based on Neural Network
}

\author{
Liu Jiashuo
}

Department of Electrical Engineering, North China Electric Power University, Baoding, China

Keywords: Power system, Reliability assessment, Rough set, Artificial neural network

\begin{abstract}
The number of components states combination and the power flow calculation are main causes producing "computation catastrophe" of reliability assessment calculation about composite generation and transmission systems. The input variables of artificial neural network are reduced, learning samples are extracted, stochastic events are roughly classified, a probable rule set about the relation between stochastic event classes and system states are draw out by means of rough set methods. A contingency pattern identification mode-Rough set and Neural Network (RNN), is presented. Furthermore, a power system reliability evaluation algorithm based on RNN is put forward for increasing the calculation speed of reliability assessment. The numerical experiments for reliability testing system s show the correctness, feasibility and usefulness of the presented method.
\end{abstract}

\section{Introduction}

When evaluating the reliability of power generation and transmission system, every incident should be analyzed and calculated [1]. The common methods are net flow method, direct current method and alternating current flow method. These methods have different advantages in terms of calculation time and accuracy[2-3]. Among them, the alternating current flow method considers the factors comprehensively and can reflect the actual situation of the power system, so the calculation precision is high. However, when the power system is large in scale, the component states will appear "combinatorial explosion", and the power flow calculation will be more laborious and often face the problem of "calculation disaster" [4]. In recent years, soft computing methods that deal with non-structural problems, such as fuzzy logic, artificial neural networks and genetic algorithms, have been widely used by human. Due to the limitations of cognition and the changing environment, based on dual logic, the precision, rigor and clarity are the main features [5]. The exact and fixed algorithm is used to solve the hard computing of the model. The problems of high dimensionality, nonlinearity, uncertainty and time variability can no longer meet the actual needs.

In this paper, the rough set and the artificial neural network are strongly and weakly coupled. The rough set of data analysis capability is used to filter the input variables of the neural network under the data-driven, refine the sample, classify the accidental events in the power system and extract the approximate relationship between the event class and the system state. Thus, based on the rough neural network model (RNN), the power system reliability assessment algorithm is established, which can improve the occasional event failure pattern recognition efficiency and reduce "Calculate Disaster" in power system reliability assessment calculation.

\section{Rough Neural Network}

The Basic Idea. Set the transmission and distribution system consists of $q$ components, $x_{i}(i=1,2, \cdots, q)$ indicates the status of the $i$ th component, $x_{i}=0$ means that element $i$ is in the DOWN state, $x_{i}=1$ means that element $i$ is in the UP state. Suppose $x=\left(x_{1}, x_{2}, \cdots, x_{q}\right)^{T}$, then $x$ represents an accidental event of the system, and a set of all contingencies is denoted as $X$. The system state variables is defined as $y$. When the system has only two states, $y=1$ is used to show the system failure, and $y=0$ is applied to indicate that the system is normal. Obviously, $y$ is a 
function of incidental event $x$, namely $y=I_{f}(x)$. So the basic calculation formula of reliability index is:

$$
E(F)=\sum_{x \in X} I_{f}(x) F(x) P(x)
$$

The problem of determining the mapping relationship between $x$ and $y$ can be regarded as a pattern recognition problem. Therefore, this paper uses artificial neural network to realize pattern recognition. The structure and some parameters of neural network can be determined by using the data analysis ability of rough set. Through reducing the input variables, refining samples, rough classification system is used to access to class features and extract the relationship between the rough set of rules. based on RBF neural network structure, the rough neural network (RNN) incident pattern recognition model is established to realize function mapping $y=I_{f}(x)$.

Build Rough Neural Network Model. For the relational data model of rough set $S=\langle U, C \cup D\rangle$, suppose the set of condition attributes as $C=\left\{x_{1}, x_{2}, x \cdots x_{q}\right\}$, the set of decision attributes as $D=\{y\}$. Thus, the incident $x$ and its corresponding system state $y$ constitute the objects in the relational data model $u=(x, y)$, and all the objects form the universe $U$. The classification of $U$ with decision attributes is denoted as $U / D=\left\{D_{0}, D_{1}\right\}$, where $D_{j}(j=0,1)$ is the set of objects representing the decision attribute value $j$.

Through the reduction of the condition attributes of the rough set, the components that are more important to system reliability are selected to realize the classification of contingencies. Conditional attribute reduction means that the minimum attribute set does not contain redundant condition attributes and ensure the correct classification. According to the nature of the dependency $\gamma_{C}(D)$ of decision attributes on conditional attributes, proceeding from the largest subset of first order dependencies, depth search is performed to obtain the set of reductions for $C$, denoted as $C^{*}=\left\{x_{i_{1}}, x_{i_{2}}, \cdots, x_{i_{r}}\right\}$. The categorization of $U$ by conditional attribute set $C^{*}$ is recorded as $U / C^{*}=\left\{C_{1}, C_{2}, \cdots, C_{L}\right\}$. The set of generalized rules between conditional attribute and decision attribute as shown in Figure 1 and Formula (2).

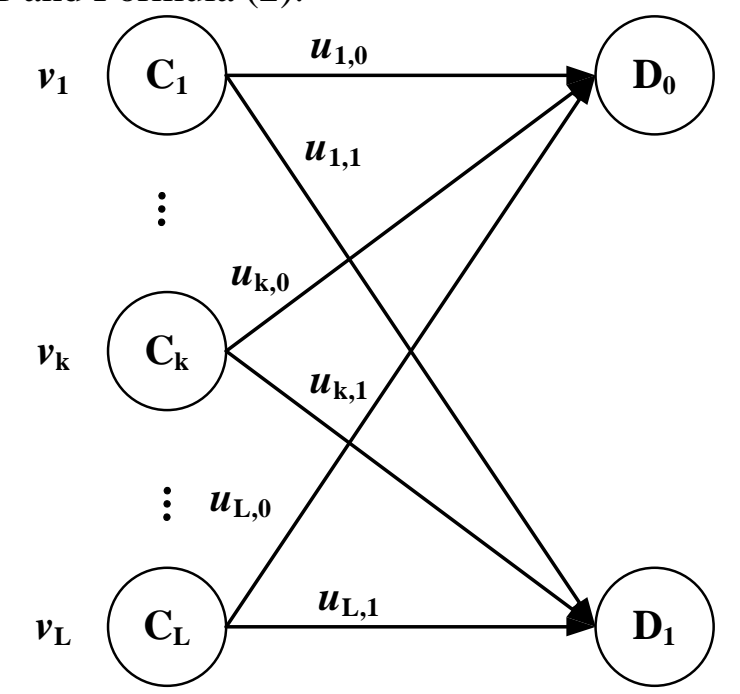

Figure 1. Simplified rules diagram

$$
W=\left\{r_{k j}: \operatorname{Des}_{C^{*}}\left(C_{k}\right) \rightarrow \operatorname{Des}_{D}\left(D_{j}\right),\left(v_{k}, u_{k, j}\right) k=1,2, \cdots, L ; j=0,1\right\}
$$

Where the component corresponding to the condition attribute $x_{i_{1}}, x_{i_{2}}, \cdots, x_{i_{r}}$ in $C^{*}$ is an important component of the system, $C_{k}(k=1,2, \cdots, L)$ is the set of objects in $U$ with the same attribute features (denoted as $\operatorname{Des}_{C^{*}}\left(C_{i}\right)$ ) in the attribute subset $C^{*}$, and the projection in the condition attribute set $C$ forms an incident class. $v_{k}$ represents the equivalence class $C_{k}$ or the 
corresponding event class in the relational data model coverage, the greater the coverage that the possibility of such events appear more. An accidental event may cause the system a variety of states, $u_{k, j}$ indicates that the corresponding event class of $C_{k}$ causes system appearing the credibility of the state.

The generalized rule sets between condition attributes and decision attributes reflect the generalized relationship between event classes and system states. The RBF network constructed by this method is called rough neural network. The network's input variables are chosen as $x_{i_{1}}, x_{i_{2}}, \cdots, x_{i_{r}}$ in the reduction set $C^{*}$. The input and output of each neuron in the input layer are equal. Set $x=\left(x_{i_{1}}, x_{i_{2}}, \cdots, x_{i_{r}}\right)^{T}$, the input variables of each neuron in the hidden layer are $x$, the number of hidden layer neurons is the elements number $L$ in the equivalent class $U / C^{*}=\left\{C_{1}, C_{2}, \cdots, C_{L}\right\}$, and the relationship between the hidden layer and the output layer is determined by the generalized rule set $W$. The activation function of neurons is Gauss function:

$$
y_{k}=\exp \left(-\frac{\left\|x-\operatorname{Des}_{C^{*}}\left(C_{k}\right)\right\|^{2}}{\sigma_{k}^{2}}\right), \quad k=1,2, \cdots, L
$$

Set

$$
\lambda_{k}=\frac{y_{k} v_{k}}{\sum_{i=1}^{L} y_{i} v_{i}}, \quad k=1,2, \cdots, L
$$

Where represents confidence level of $C_{k}$ that accidental event $x$ belongs. The output of the output layer is:

$$
z_{j}=z_{j}(x)=\sum_{k=1}^{L} \lambda_{k} u_{k, j}
$$

Where indicates the probability that contingency $x$ is in the $j$ th state of the system. Apparently, $0 \leq z_{j} \leq 1, z_{0}+z_{1}=1$. Suppose the expected output of incident $x$ is:

$e_{j}(x)=\left\{\begin{array}{l}1, x \text { put the system in } i \text { state } \\ 0, x \text { put the system in } j \text { state }\end{array}\right.$

The error function is chosen as:

$$
Q\left(\sigma_{1}, \sigma_{2}, \cdots, \sigma_{L}\right)=\sum_{x \in \tilde{G}} \sum_{i=0}^{1}\left(e_{i}(x)-z_{i}(x)\right)^{2}
$$

Where $\tilde{G}$ is the projection of $U$ in the $x$-direction, namely a set of learning samples for incidental events.

Reliability Assessment Algorithm based on RNN. The RNN embedded in the transmission and distribution system reliability assessment calculation can be formed as shown in Figure 1.

\section{Case Study}

The reliability test system of RBTS6 and IEEE-RTS79 is verified based on RNN transmission combined system reliability assessment method.

Table 1 shows the classification results of the accidental events of the RBTS test system by using rough sets with 6 reduction accuracies $(0.0001,0.0005,0.001,0.0000,0.01,0.05)$. The calculation results in Table 1 show that the number of input variables and the number of events are decreasing with the reduction of reduction precision. This is because the lower the reduction accuracy, the smaller the reduction set, the more rough the classification of events, the higher the similarity between occasions, easy clustering, and thus reducing the number of events. In fact, the reduction and classification process is a screening process for components that have a major impact on system reliability. For the RBTS test system, when the reduction accuracy is 0.05 , the input variables 
screened, that is, some components of the system are shown in Table 2. These elements are all very important components of the system reliability. For example, once the fault of line L9 will cause system breakdown, all the $20 \mathrm{MW}$ load of node 6 will lose its power supply while generator bus \# 2G1 bus \# 1G1 and bus \# 1G2 is the largest unit in the system capacity and the worst reliability of the unit.

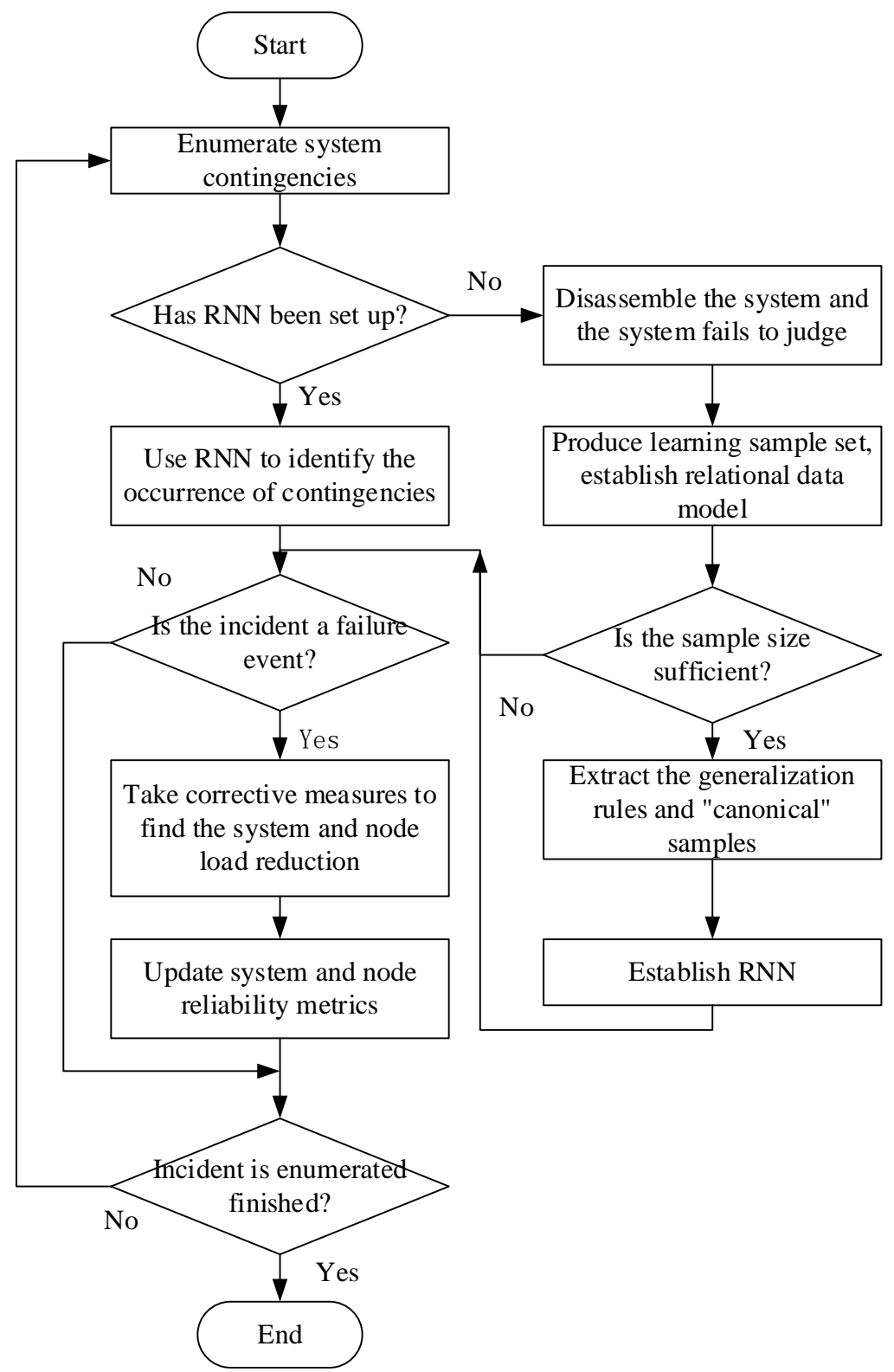

Figure 2. RNN based reliability calculation process

Table 1. Rough sets RBTS test system incident classification results

\begin{tabular}{cccc}
\hline Reduction accuracy & $\begin{array}{c}\text { Number of input } \\
\text { variables }\end{array}$ & Number of events & Experience in error \\
\hline 0.0001 & 16 & 79 & 40.2583 \\
0.0005 & 15 & 68 & 35.3484 \\
0.0010 & 12 & 41 & 21.7893 \\
0.0050 & 11 & 39 & 23.3417 \\
0.0100 & 9 & 25 & 36.5740 \\
0.0500 & 8 & 22 & 33.9727 \\
\hline
\end{tabular}


Table 2 Components screened by rough set (RBTS system)

\begin{tabular}{ccc}
\hline Component name & Component capacity / MW & Component invalidation \\
\hline LineL9 & 71 & 0.00114 \\
Generator \#2G1 & 40 & 0.02 \\
Generator \#1G1 & 40 & 0.03 \\
Generator \#1G2 & 40 & 0.03 \\
Line L1 & 85 & 0.0017 \\
Line L2 & 85 & 0.0017 \\
Generator \#2G2 & 20 & 0.15 \\
Generator \#2G3 & 20 & 0.15 \\
\hline
\end{tabular}

The reliability index of IEEE - RTS79 test system is calculated by using RNN - based reliability assessment algorithm. The calculation results and the results of other methods are shown in Table 3 . Using RNN algorithm to calculate the reliability of IEEE - RTS79 system with different reduction precision, the results show that with the reduction of precision, the classification of rough sets is more rough, and the calculation accuracy of EENS is also declining, but at the same time, time also showed a clear downward trend. This shows that the appropriate choice of reduction accuracy can significantly reduce the calculation time with reasonable calculation accuracy.

Table 3. IEEE - RTS79 reliability test system calculation results

\begin{tabular}{cccccc}
\hline $\begin{array}{c}\text { Calculation } \\
\text { method }\end{array}$ & $\begin{array}{c}\text { Loss of load } \\
\text { probability } \\
\left(\times 10^{-2}\right)\end{array}$ & $\begin{array}{c}\text { Low battery } \\
\text { expectation } \\
\left(\times 10^{2} /\left(M W h \cdot a^{-1}\right)\right)\end{array}$ & $\begin{array}{c}\text { Load loss } \\
\text { frequency } \\
\left(\times 10^{1} /\left(\text { times } \cdot a^{-1}\right)\right)\end{array}$ & $\begin{array}{c}\text { Load loss } \\
\text { expectation } \\
\left(\times 10^{2} /\left(h \cdot a^{-1}\right)\right)\end{array}$ & Time/s \\
\hline $\begin{array}{c}\text { Net flow } \\
\text { method }\end{array}$ & 9.371 & 12.200 & 6.537 & 8.209 & 2 \\
DC method & 7.536 & 12.400 & 4.808 & 6.594 & 698 \\
RNN & 8.467 & 9.164 & 5.461 & 7.417 & 169 \\
\hline
\end{tabular}

\section{Conclusion}

Calculating power flow for every contingency is one of the main reasons for "calculating disaster". In this paper, RNN for pattern recognition of incidental events is proposed from the viewpoint of reducing the number of tidal flows and increasing the pattern recognition efficiency of accidental events, through the strong and weak coupling between rough sets and artificial neural networks. By rough set data analysis method, the input variables are reduced, the learning sample and the approximate relationship between the event class and the system state are refined, and then the neural network is established, which makes the neural network concept of physics clear, interpretable, and of computational speed. The reliability evaluation algorithm based on RNN and the reliability calculation of IEEE - RTS79 system verify the correctness, feasibility and validity of the proposed algorithm and provide a new solution to the "computing disaster" of reliability evaluation way.

\section{References}

[1] Dai Z, Li Z, Jiao Y, et al. Reliability assessment based on BP neural network for relay protection system with a few failure data samples[J]. Electric Power Automation Equipment, 2014, 34(11): 129-134.

[2] Ebrahimpour R, Abharian E K, Moussavi S Z, et al. Transient Stability Assessment of a Power System by Mixture of Experts[J]. International Journal of Engineering, 2013, 4(1):93-104. 
[3] Zhong B. Research on Soft Computing Models for Reliability Assessment of Bulk Power Systems[J]. Transactions of China Electrotechnical Society, 2005.

[4] Li C, Qing G, Li P, et al. Operational Risk Assessment of Distribution Network Equipment Based on Rough Set and D-S Evidence Theory[J]. Journal of Applied Mathematics, 2013, 1: 1-7.

[5] Patowary P, Goyal N K. Security monitoring and assessment of an electric power system[J]. International Journal of Performability Engineering, 2014, 10(3): 273-280. 